\title{
Effect of ramipril on renal function in patients with intermittent claudication
}

\author{
Simon D Hobbs' \\ Martin W Claridge' \\ Antonius BM Wilmink' \\ Donald J Adam' \\ Mark E Thomas ${ }^{2}$ \\ Andrew W Bradbury' \\ 'University Department of Vascular \\ Surgery and ${ }^{2}$ Department of \\ Nephrology, Heart of England NHS \\ Trust (Teaching), Birmingham, UK
}

Correspondence: Andrew W Bradbury

University Department of Vascular

Surgery, Research Institute,

Lincoln House, Birmingham Heartlands

and Solihull NHS Trust (Teaching),

Bordesley Green East, Birmingham,

B9 5SS, UK

Tel/fax +44 I2I 424 I633

Email andrew.bradbury@heartsol.wmids. nhs.uk

\begin{abstract}
Background: The Heart Outcomes Prevention Study (HOPE) demonstrated that ramipril resulted in a blood-pressure-independent $25 \%$ reduction in cardiovascular events in patients with peripheral arterial disease (PAD). Despite this, general practitioners and vascular surgeons remain reluctant to prescribe ACE inhibitors in this group of patients because of concerns about renal artery stenosis (RAS). We aimed to define the effect of ramipril on renal function in patients with intermittent claudication (IC).
\end{abstract}

Methods and Results: Of 132 unselected patients with IC entering the study 78 (59\%) were excluded due to: current ACE inhibitor use (38\%), renal impairment (serum creatinine above normal range) (15\%), known severe RAS (1\%) or unwillingness to participate $(5 \%)$. The remaining 54 patients were titrated to $10 \mathrm{mg}$ ramipril and renal function was monitored at 1,5 , and 12 weeks. Treatment was discontinued during titration in 5 patients due to symptoms (3) or lack of compliance (2). In the remainder, median [IQR] serum creatinine increased (94 [85.8-103.3] to 98 [88.0-106.5] $\mu \mathrm{mol} / \mathrm{L}, \mathrm{p} \leq 0.001)$ and median [IQR] GFR decreased (71.5 [64.6-82.3] to $68.7[59.8-74.7] \mathrm{mL} / \mathrm{min}$ per $1.73 \mathrm{~m}^{2}, \mathrm{p} \leq 0.001$ ) between baseline and 5 weeks. These changes were not considered clinically significant. By 12 weeks these values had returned almost to baseline (Cr $95.5[88.0-103.25] \mu \mathrm{mol} / \mathrm{L}$, GFR 71.8 [65.3-77.4] mL/min). No patient had a serum creatinine rise $>30 \%$.

Conclusion: Most of patients with IC and a normal serum creatinine can be safely commenced on ramipril provided they are screened, titrated and monitored as described above. Studies in patients with borderline renal impairment (serum creatinine up to $30 \%$ above baseline) are on-going.

Keywords: peripheral arterial disease, ramipril, renal function, intermittent claudication

\section{Introduction}

Best medical therapy (BMT) is the mainstay of treatment for patients with peripheral arterial disease (PAD) with endovascular or surgical intervention reserved for those who remain unacceptably symptomatic or have life and/or limb-threatening complications (Burns et al 2003).

In addition to smoking cessation therapies, hypertension and diabetic control, and antiplatelet and lipid lowering medication, the publication of the Heart Outcomes Prevention Evaluation (HOPE) study has provided level 1 evidence to support the use of ramipril for secondary cardiac prevention in patients with PAD (The Heart Outcomes Prevention Evaluation Study Investigators 2000).

The HOPE study recruited patients with a history of cardiovascular disease (including a cohort of 4051 (44\%) patients with PAD) or a history of diabetes plus at least one other risk factor and randomized them to receive ramipril (titrated up to $10 \mathrm{mg}$ ) or placebo. At a mean follow-up of 4.5 years, ramipril significantly reduced the composite end-point (myocardial infarction, stroke, or death from cardiovascular causes) by $25 \%$. This reduction in risk was at least as large as that observed for treatment with aspirin and lipid-lowering agents (Heart Protection Study Collaborative Group 2002; 


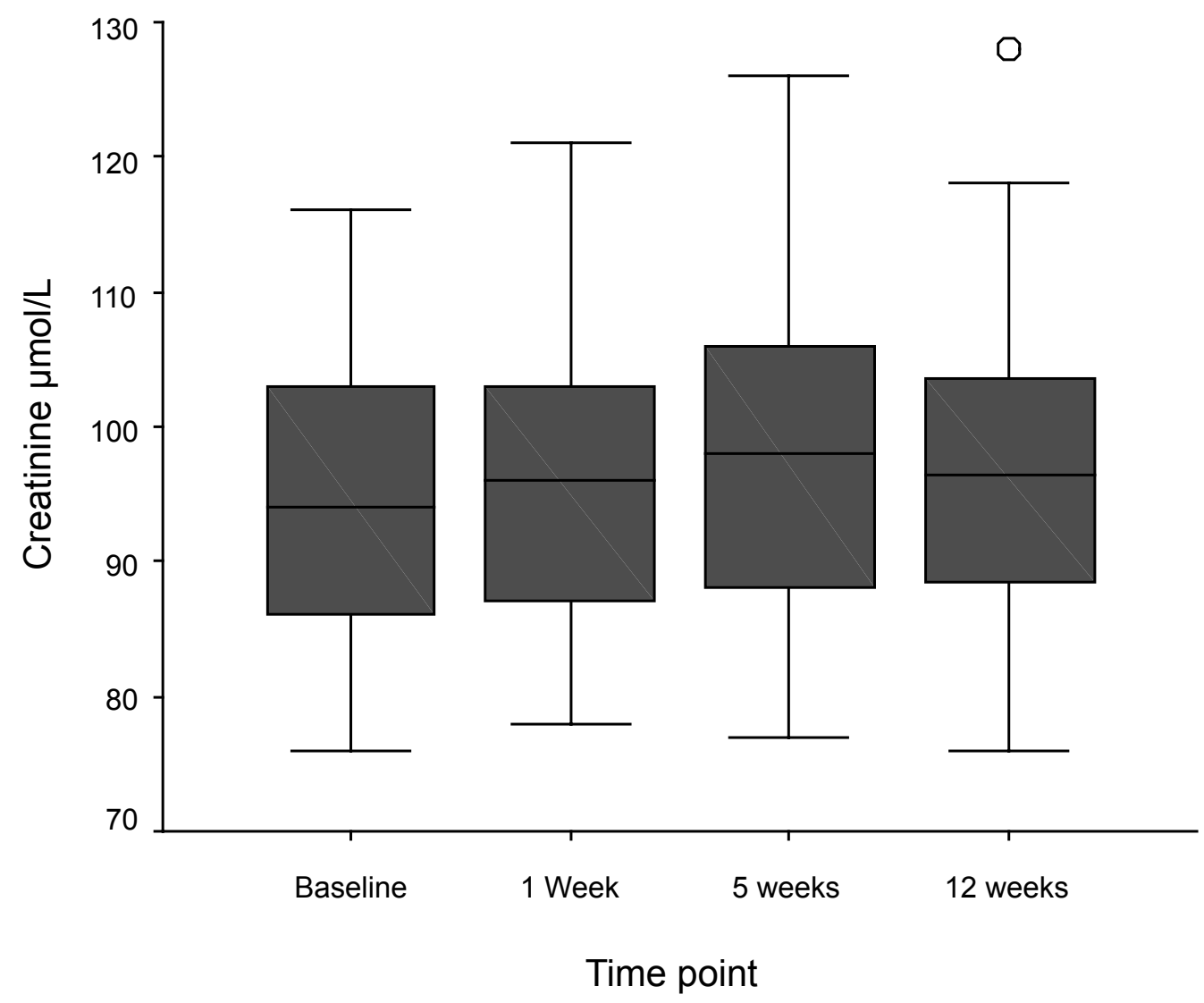

Figure I Boxplots of change in serum creatinine in response to initiation of ramipril. Patients are commenced on ramipril $2.5 \mathrm{mg}$ for I week, increased to 5 mg for 3 weeks, and then maintained on $10 \mathrm{mg}$. Compared with baseline, I week $p=0.015,5$ weeks $p \leq 0.001$, I 2 weeks $p=0.003$, Wilcoxon signed ranks).

Peripheral Arterial Diseases Antiplatelet Consensus Group 2003) and was independent of entry blood pressure.

Although the HOPE study indicated that the benefits of ACE inhibition in patients with PAD outweigh the risks, vascular surgeons remain wary of the small risk of precipitating acute renal failure in patients with hemodynamically significant renal artery stenosis (RAS) (Pillay et al 2002). The aim of this study was to evaluate in detail for the first time, the effect of ramipril titration on renal function in a cohort of surgical PAD patients presenting with intermittent claudication, in the expectation that if safety could be demonstrated then this would change vascular surgeons' practice.

\section{Patients and methods}

Local ethical approval was obtained and written informed consent was obtained from all patients. Patients, over the age of 55 years, attending the vascular surgical outpatient department of a large teaching hospital with a diagnosis intermittent claudication (IC) based on structured history (Leng and Fowkes 1992) and ankle brachial pressure index (ABPI) entered the study. Patients were excluded if they were currently receiving an $\mathrm{ACE}$ inhibitor or angiotensin receptor blocker, had abnormal baseline renal function (serum creatinine $>120 \mu \mathrm{mol} / \mathrm{L}$ males, $>110 \mu \mathrm{mol} / \mathrm{L}$ females), a history of severe renal artery stenosis (RAS), or were unwilling to participate. Patients who fulfilled the entry criteria were commenced on ramipril (Tritace ${ }^{\circledR}$ titration pack, Aventis Pharma UK), starting at $2.5 \mathrm{mg}$ for 1 week, increased to $5 \mathrm{mg}$ for 3 weeks and then further increased to the maintenance dose of $10 \mathrm{mg}$. Renal function was monitored by means of a blood sample collected after 1, 5, and 12 weeks and glomerular filtration rate (adjusted to a body surface area of $1.73 \mathrm{~m}^{2}$ ) was estimated by means of the Modification of Diet in Renal Disease (MDRD) Equation 7 (Levey et al 1999). The MDRD equation 7 requires serum creatinine, urea, and albumin values and it factors for the patient's age, sex, and race. It has largely superseded the Cockcroft-Gault equation, as it has improved accuracy and does not require knowledge of the patient's weight (Nation Kidney Foundation 2007). Ramipril was to be discontinued if the patient's serum creatinine rose greater than $30 \%$ from their baseline value or if serum potassium rose $>5.9 \mathrm{mmol} / \mathrm{L}$, in line with our 
recently published treatment algorithm (Hobbs et al 2004). Ramipril was also discontinued if the subject encountered unacceptable side effects.

Statistical analysis was performed using the SPSS 11.0 for windows statistical package (SPSS Inc, Chicago, Ill). The Wilcoxon signed ranks test was used to analyze changes in creatinine and glomerular filtration rate (GFR) over time.

\section{Results}

One hundred and thirty-two consecutive patients (median age [interquartile range, IQR], 71 [62.25-78] years; 89 male) with IC were screened for study entry. Of these, 78 (59\%) were excluded from the study for the following reasons. 38 $(29 \%)$ patients were already receiving an ACE inhibitor, 11 $(8 \%)$ were receiving an angiotensin receptor blocker, and 1 patient was receiving both an ACE inhibitor and an angiotensin receptor blocker. 20 (15\%) patients had biochemical evidence of renal impairment (median serum creatinine [IQR] 129 [124-139] $\mu \mathrm{mol} / \mathrm{L})$. Of the remainder, 1 patient had a history of RAS and 7 (5\%) patients were unwilling to participate.

Fifty-four (41\%) patients (Table 1) fulfilled the entry criteria and were commenced on ramipril. ACE inhibition was discontinued during titration (within 5 weeks of start) due to adverse events in 3 patients ( 1 had exacerbation of angina and 2 reported dizzy episodes) and lack of compliance with monitoring blood tests in 2 . A further 5 patients were discontinued after titration to $10 \mathrm{mg}$, but before 12 weeks. Three of these patients had adverse events (one each of cough, headaches, and arthralgia) and 2 failed to comply with monitoring blood tests. All of the four patients who were non-compliant with follow-up were contacted by telephone and none reported adverse events.

During titration to $10 \mathrm{mg}$ ramipril, a small but statistically significant rise in serum creatinine (median [IQR] creatinine 94 [85.8-103.3] $\mu \mathrm{mol} / \mathrm{L}$ to $98[88.0-106.5] \mu \mathrm{mol} / \mathrm{L}$, $\mathrm{p} \leq 0.001$ ) and fall in GFR (median [IQR] GFR 71.5 [64.6-82.3] mL/min per $1.73 \mathrm{~m}^{2}$ to 68.7 [59.8-74.7] mL/ min per $1.73 \mathrm{~m}^{2}, \mathrm{p} \leq 0.001$ ) was observed. No patient had a serum creatinine rise greater than $30 \%$ from their baseline value and no patient had a serum potassium rise greater than $5.9 \mathrm{mmol} / \mathrm{L}$. By 12 weeks renal function had almost returned to baseline levels (median creatinine [IQR] 96.5 [88.3-103.8] $\mu \mathrm{mol} / \mathrm{L}, \mathrm{p}=0.003$ and median GFR [IQR] 69.8 [65.0-76.9] $\mathrm{mL} / \mathrm{min}$ per $\left.1.73 \mathrm{~m}^{2}, \mathrm{p}=0.015\right)$.

\section{Discussion}

This study demonstrates that using our treatment algorithm (Hobbs et al 2004), most patients presenting to vascular
Table I Demographic details of patients commenced on ramipril

\begin{tabular}{ll}
\hline Variable & \\
\hline Number of subjects & 54 \\
Median age [IQR] & $67.5[59-76]$ \\
Sex (M:F ratio) & $3.5: 1$ \\
Ischemic heart disease & \\
$\quad$ Myocardial infarction/angina & 8 \\
$\quad$ Coronary artery bypass graft & 1 \\
Cerebrovascular disease & \\
$\quad$ Cerebrovascular accident & 1 \\
$\quad$ Transient ischemic attack & 0 \\
Diabetes mellitus & 8 \\
Hypertension & 27 \\
Medication & \\
$\quad$ Antiplatelet agent & \\
$\quad$ Statin & 54 \\
$\quad$ Antihypertensive & 53 \\
$\quad$ Insulin & 25 \\
Oral hypoglycemic & 2 \\
Mean ABPI (range) & 1
\end{tabular}

Abbreviations: $\mathrm{ABPI}$, ankle brachial pressure index; IQR, interquartile range.

surgeons with a confirmed diagnosis of IC and normal renal function can be titrated up to the evidence-based dose $(10 \mathrm{mg})$ of ramipril with no deleterious effect to renal function. These novel data also suggest that the perceived risk of RAS in such patients exceeds the actual risk and in the presence of a normal baseline creatinine no further assessment of renal function or imaging of the renal arteries is required prior to commencing ramipril. Although we have demonstrated short-term safety, it is important to remain vigilant as intercurrent illness and dehydration may precipitate impaired renal function in subjects receiving ACE inhibitors.

Level 1 evidence exists to support the use of ramipril for secondary cardiac prevention in patients with PAD manifest by: previous limb bypass surgery or percutaneous angioplasty; previous limb or foot amputation; history of intermittent claudication with ankle; arm blood pressure $<0.8$ in at least one side; or a significant stenosis $(>50 \%)$ documented by angiography (The Heart Outcomes Prevention Evaluation Study Investigators 1996). The recently published Inter-Society Consensus for the management of Peripheral Vascular Disease (TASC II) guidelines recommend that ACE inhibitors should be considered as first line antihypertensive treatment to reduce the risk of cardiovascular events (Inter-Society Consensus for the Management of Peripheral Arterial Disease 2007).

Despite this evidence, a large number of patients with PAD remain untreated. Part of the reason for this is that vascular surgeons remain cautious about commencing ACE 


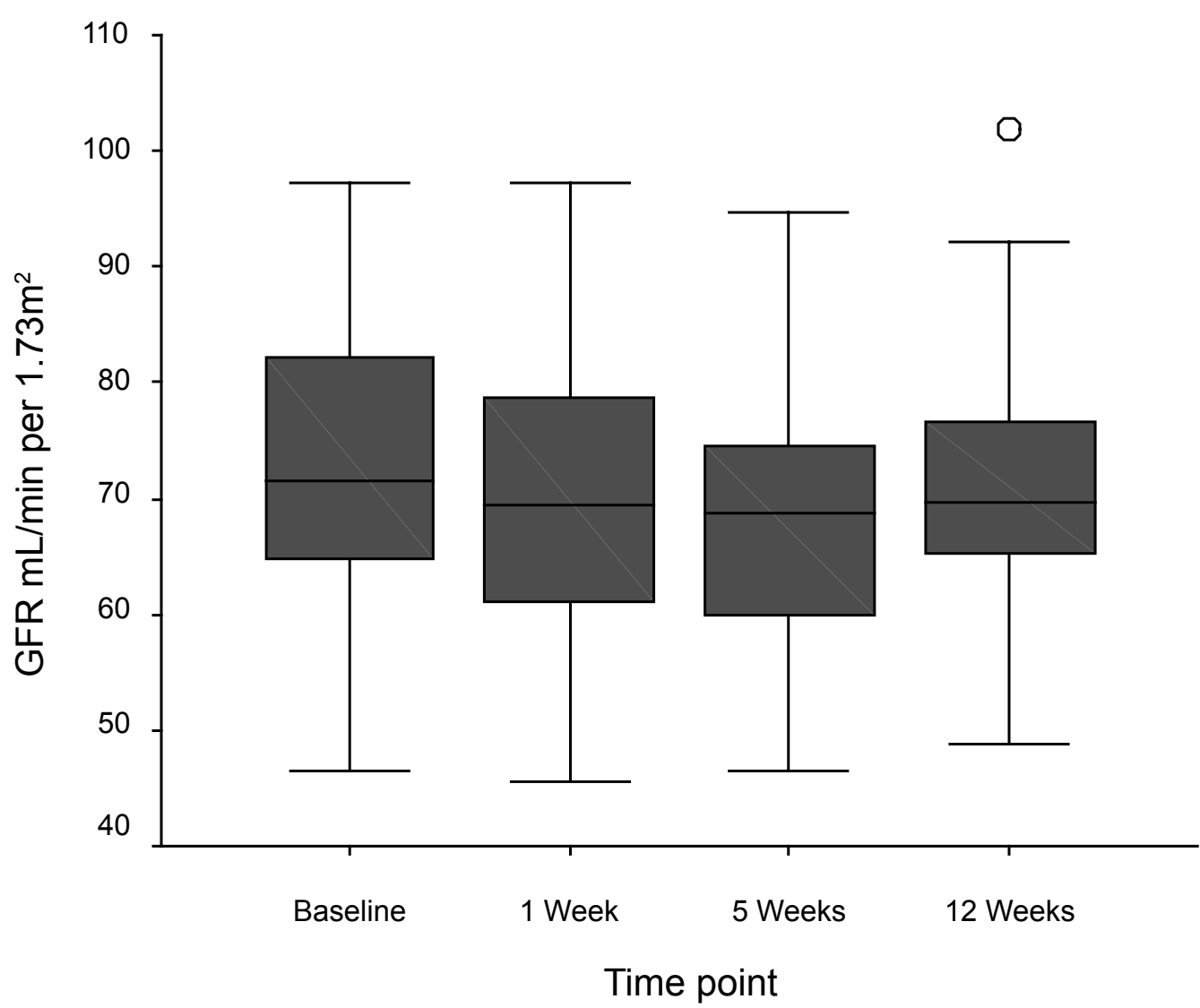

Figure 2 Boxplots of change in glomerular filtration rate in response to initiation of ramipril. Patients are commenced on ramipril $2.5 \mathrm{mg}$ for I week, increased to $5 \mathrm{mg}$ for 3 weeks, and then maintained on $10 \mathrm{mg}$. Compared with baseline, I week $p=0.009,5$ weeks $p \leq 0.001,12$ weeks $p=0.015$, Wilcoxon signed ranks).

inhibitors in a patient population that has a high perceived risk of RAS. Although the incidence of RAS in patients with PAD is not well established, it has been reported to be between $20 \%$ and $50 \%$ in angiographic series, with $12 \%$ to $24 \%$ of subjects having bilateral RAS (Choudhri et al 1990; Salmon and Brown 1990; Missouris et al 1994; Iglesias et al 2000). In practice, however, the incidence of RAS in our less selected group of patients with PAD is likely to be lower than this, since most patients with PAD do not have disease severe enough to warrant angiography and are likely to have correspondingly less advanced atheromatous renovascular disease.

In this study we have demonstrated a clinically insignificant, but statistically significant, rise in serum creatinine and fall in GFR in response to titrating ramipril up to $10 \mathrm{mg}$ over a 5 -week period. However, these levels return virtually to baseline by 12 weeks and no patient had a serum creatinine rise of greater than $30 \%$ or developed hyperkalemia. Interestingly, in patients with pre-existing chronic renal insufficiency, an early rise in serum creatinine of up to $30 \%$ with ACE inhibition is associated with long-term renal protection (Bakris and Weir
2000). The mechanism is unknown, but it is thought that "remnant" or surviving nephrons may be subject to deleterious hyperfiltration. ACE inhibition, by causing vasodilatation of the efferent arteriole, may reduce this potentially deleterious hyperfiltration. The early fall in GFR may, therefore, herald a longer-term protective effect.

It is of note that $30 \%$ of patients screened for entry into this study were already receiving ACE inhibitors, predominantly for the treatment of hypertension or as secondary prevention following myocardial infarction. A further 11 patients were receiving an angiotensin receptor blocker for treatment of their hypertension. It is not yet known whether angiotensin receptor blockers confer the cardio protective benefits for this patient group that are evident with ramipril.

Ramipril is currently available generically at a cost of $£ 14.24$ for a 28 -tablet pack $(10 \mathrm{mg})$. This compares very favorably with other drugs used for cardiovascular risk reduction (eg, simvastatin $40 \mathrm{mg}-£ 29.69$ for a 28-tablet pack). Furthermore, several economic studies assessing the benefits of ramipril based on data from the HOPE study have all concluded that ramipril is highly cost effective 
in the treatment of patients at high risk of cardiovascular events and that its use is likely to represent an efficient use of resources (Bjorholt et al 2002; Lamy et al 2003; Smith et al 2003).

Although the current results show that most claudicants presenting to a vascular surgeon can safely be commenced on ACE inhibitors, the results may not be applicable to all subjects with IC. Firstly, 15\% of patients had abnormal renal function at baseline and were therefore excluded from this present study. The median serum creatinine for these patients, however, was only moderately elevated (129 [IQR, 124-139] $\mu \mathrm{mol} / \mathrm{L}$ ) and as such most could probably be safely commenced on ramipril, although this will need to be confirmed in a future study. Secondly, $11 \%$ of patients commenced on ramipril had to discontinue the drug due to the development of recognized side effects. Half of these side effects occurred while on the higher doses of the drug and potentially, therefore, these patients could have been maintained on a lower dose of ramipril. Lastly, a small proportion of patients were excluded as they were unwilling to commence a new tablet or unable to attend for surveillance blood tests. It may not be possible to extrapolate these results to subjects with more severe forms of PAD (eg, critical limb ischemia).

In conclusion, therefore, this study has demonstrated for the first time that most patients presenting to vascular surgeons with IC can be safely commenced on ramipril provided the cautions and algorithm described above are adhered to. It is hoped that these novel data will change surgeons' practice so that greater numbers of PAD patients will enjoy the very considerable benefits of this evidencebased therapy.

\section{Disclosures}

Aventis Pharma UK supplied the Tritace ${ }^{\circledR}$ titration packs free of charge but had no influence on the study design, interpretation of the data, or preparation of the manuscript.

\section{References}

Bakris GL, Weir MR. 2000. Angiotensin-converting enzyme inhibitorassociated elevations in serum creatinine: Is this a cause for concern? Arch Int Med, 160:685-93.
Bjorholt I, Andersson FL, Kahan T, et al. 2002. The cost-effectiveness of ramipril in the treatment of patients at high risk of cardiovascular events: a Swedish sub-study to the HOPE study. J Int Med, 251:508-17.

Burns P, Gough S, Bradbury AW. 2003. Management of peripheral arterial disease in primary care. $B M J, 326: 584-8$.

Choudhri AH, Cleland JGF, Rowlands PC, et al. 1990. Unsuspected renal artery stenosis in peripheral vascular disease. BMJ, 301:1197-8.

Heart Protection Study Collaborative Group. 2002. MRC/BHF Heart Protection Study of cholesterol lowering with simvastatin in 20,536 high-risk individuals: a randomised placebo-controlled trial. Lancet, 360:7-22.

Hobbs SD, Thomas ME, Bradbury AW. 2004. Manipulation of the renin angiotensin system in peripheral arterial disease. Eur J Vasc Endovasc Surg, 28:573-82.

Iglesias JI, Hamburger RJ, Feldman L, et al. 2000. The natural history of incidental renal artery stenosis in patients with aortoiliac vascular disease. Am J Med, 109:642-7.

Inter-Society Consensus for the Management of Peripheral Arterial Disease (TASC II). 2007. Eur J Vasc Endovasc Surg, 33(Suppl 1).

Lamy A, Yusuf S, Pogue J, et al. 2003. Cost implications of the use of ramipril in high-risk patients based on the Heart Outcomes Prevention Evaluation (HOPE) study. Circulation, 107:960-5.

Leng GC, Fowkes FGR. 1992. The Edinburgh Claudication Questionnaire: an improved version of the WHO / Rose Questionnaire for use in epidemiological surveys. J Clin Epidemiol, 45:1101-9.

Levey AS, Bosch JP, Breyer J, et al. 1999. A more accurate method to estimate glomerular filtration rate from serum creatinine: a new prediction equation. Modification of Diet in Renal Disease Study Group. Ann Intern Med, 130:461-70.

Missouris CG, Buckenham T, Cappuccio FP, et al. 1994. Renal artery stenosis: A common and important problem in patients with peripheral vascular disease. Am J Med, 96:10-14.

National Kidney Foundation K/DOQI Guidelines. 2007. Evaluation of laboratory measurements for clinical assessment of kidney disease. Guideline 4. Estimation of GFR [online]. [Accessed 18 July 2007]. URL: http://www.kidney.org/professionals/doqi/kdoqi/p5_lab_g4.htm.

Peripheral Arterial Diseases Antiplatelet Consensus Group. 2003. Antiplatelet therapy in peripheral arterial disease. Consensus statement. Eur $J$ Vasc Endovasc Surg, 26:1-16.

Pillay WR, Kan YM, Crinnon JN, et al. 2002. for the Joint Vascular Research Group. Prospective multicentre study of the natural history of atherosclerotic renal artery disease in patients with peripheral vascular disease. Br J Surg, 89:737-40.

Salmon P, Brown MA. 1990. Renal artery stenosis and peripheral arterial disease: implications for ACE inhibitor therapy. Lancet, 336:321.

Smith MG, Neville AM, Middleton JC. 2003. Clinical and economic benefits of ramipril: an Australian analysis of the HOPE study. Int Med $J, 33: 414-19$

The Heart Outcomes Prevention Evaluation Study Investigators. 1996. The HOPE (Heart Outcomes Prevention Evaluation) study: The design of a large, simple randomised trial of an angiotensin-converting enzyme inhibitor (ramipril) and vitamin $\mathrm{E}$ in patients at high risk of cardiovascular events. Can J Cardiol, 12:127-37.

The Heart Outcomes Prevention Evaluation Study Investigators. 2000. Effects of an angiotensin-converting-enzyme inhibitor, Ramipril, on cardiovascular events in high-risk patients. $N$ Engl J Med, 342:145-53. 
